\title{
ТЕОРЕТИКО-ВЕРОЯТНОСТНЫЕ МЕТОДЫ И МОДЕЛИ В СИСТЕМЕ УПРАВЛЕНИЯ ИНФОРМАЦИОННО-АНАЛИТИЧЕСКОЙ ПОДГОТОВКИ СПЕЦИАЛИСТОВ МЧС РОССИИ
}

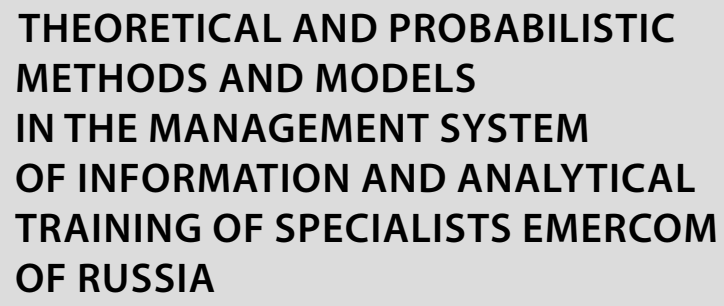

THEORETICAL AND PROBABILISTIC METHODS AND MODELS

IN THE MANAGEMENT SYSTEM

OF INFORMATION AND ANALYTICAL TRAINING OF SPECIALISTS EMERCOM OF RUSSIA

E. Trofimets

Summary. The questions of the application of theoretical and probabilistic methods and models in the system of information and analytical training of specialists of the Ministry of Emergency Situations of Russia are considered. Computer templates have been developed that allow modeling various situational decision-making tasks based on the Bernoulli scheme. The applied aspects of the approximation of a binomial distribution by a normal one are investigated.

Keywords: information and analytical training, probability theory, practice-oriented tasks.
B процессе подготовки специалистов МЧС России в рамках цифровизации образовательной среды актуально использовать на занятиях по математическим дисциплинам пакеты прикладных программ при решении практико-ориентированных задач (ПОЗ) [1-7].

В данной работе фокус внимания смещен на применение компьютерной системы Excel при изучении темы: «Применение базовых теорем теории вероятностей к решению практико-ориентированных задач».

Данная тема актуальна и значима в профессиональной деятельности инженера-аналитика системного анализа центра управления в кризисных ситуациях, а также инженера по пожарной безопасности, инженера по техносферной безопасности, юриста и других сотрудников МЧС России.

Актуальность обусловлена прогнозированием опасных факторов пожара, проведением, мониторинга пожарной обстановки, прогнозированием стихийных бедствий, ЧС техногенного характера, ЧС природного характера, оценкой ликвидации последствий стихий- трофимец Елена Николаевна

К.п.н., дочент, ФГБОУ ВО «Санкт-Петербургский университет Государственной противопожарной службы Министерства РФ по делам гражданской обороны, чрезвычайным ситуациям и ликвидации последствий стихийных бедствий» ezemifort@inbox.ru

Аннотация. Рассмотрены вопросы применения теоретико-вероятностных методов и моделей в системе информационно-аналитической подготовки специалистов МЧС России. Разработаны компьютерные шаблоны, позволяющие моделировать различные ситуационные задачи принятия решений на основе схемы Бернулли. Исследованы прикладные аспекты аппроксимации биномиального распределения нормальным.

Ключевые слова: информационно-аналитическая подготовка, теория вероятностей, практико-ориентированные задачи.

ных бедствий, предупреждение ЧС, эпидемиологической обстановкой в стране.

Математический аппарат теории вероятностей и математической статистики будет использован и в профессиональных дисциплинах: Теоретические основы электротехники и электроника; Метрология, стандартизация и сертификация; Теория информационных систем; Системный анализ, оптимизация и принятие решений; Управление в организационных системах; Исследование операций и методы оптимизации; Системы связи и оповещения; Системы инженерного и космического мониторинга; Базы данных; Антикризисное управление в чрезвычайных ситуациях; Опасные природные процессы; Опасные ситуации природного характера и защита от них; Экономика и финансы при чрезвычайных ситуациях.

Перейдем к постановке ПОЗ № 1 с различными видами ситуаций.

Ситуация 1. Точность экспресс-теста на вирус составляет $80 \%$ (20\% - ложноотрицательный результат). Тест сдали 3 заболевших человека. Какова вероятность того, что заболевание будет выявлено: 


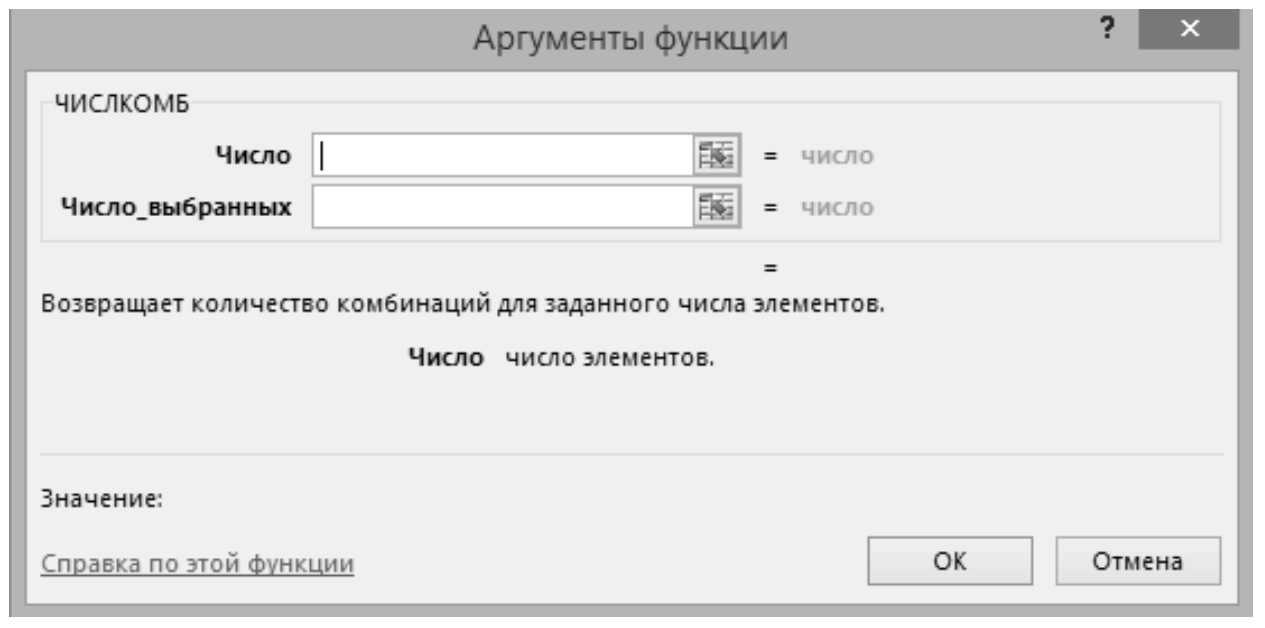

Рис. 1. Аргументы функции «ЧИСЛОКОМБ»

а) у всех заболевших; б) у двух заболевших; в) у одного заболевшего; г) не будет выявлено ни у кого.

Классическое решение ПОЗ ситуации 1 - использование теорем сложения и умножения вероятностей.

Ситуациия 2. Теперь рассмотрим эту же ПОЗ, при условии, что тестируется 100 заболевших. Для решения задачи нам потребуется рассмотреть 101-у разновидность составных событий, каждое из которых будет образовано 100 элементарными. Таким образом, при решении задачи «в лоб» трудоемкость существенно возрастает. В этом случае на помощь приходит схема испытаний или формула Бернулли.

Действительно схема испытаний Бернулли состоит в том, что проводятся независимые несколько раз повторяемые (часто многократно повторяемые) испытания. При этом каждое такое испытание приводит к одному из двух возможных исходов, называемых часто успехом и неудачей, и вероятность успеха не меняется от одного опыта к другому.

Наиболее знаком нам пример многократного подбрасывания монеты. Классические примеры некоторых постановок задач, которые также хорошо описываются схемой испытаний Бернулли.

1. Запланирован запуск шестидесяти космических аппаратов. Вероятность успешного выведения на заданную орбиту запуска каждого аппарата равна 0,99. Какова вероятность того, что не менее пятидесяти девяти космических аппаратов успешно выйдут на заданную орбиту?

2. Транспорт гибнет от попадания двух авиабомб весом по 120 кг или одной авиабомбы 200 кг. Самолет может взять авиабомбы одного типа общим весом 1200 кг. Какого типа авиабомбы вы- годнее брать, если вероятность попадания авиабомбы первого типа равна 0,08, а второго - 0,05?

Рассмотренная в ситуации 1 задача по выявлению заболевания на основе экспресс-теста также соответствует схеме испытаний Бернулли. В этом случае успехом удобнее считать выявление заболевания, хотя в жизни для человека это конечно же наступление «плохого» события, т.е., наоборот, неуспех. Поэтому ещё раз обращаю внимание, на разную смысловую нагрузку термина «успех» в теории вероятностей и в жизненных ситуациях.

Итак, приступим к решению задачи в MS Excel.

В формулу Бернулли в качестве сомножителя входит число сочетаний из $n$ элементов по $m$. Так как сочетания нами уже многократно рассматривались, предлагается для их вычисления использовать функцию чИСЛОКОМБ (категория Математические), которая имеет два аргумента (Рис. 1): Число и Число_выбранных. Для рассматриваемой ПОЗ эти аргументы имеют следующую интерпретацию:

- первый аргумент Число - число испытаний (тестирований);

- второй аргумент Число_выбранных - число «успехов» (выявленных заболеваний).

Эффективность формулы Бернулли особенно проявляется при достаточно большом числе испытаний. Рассмотрим следующую ПОЗ № 2.

ПОЗ № 2. Точность экспресс-теста на вирус составляет $80 \%$ (20\% - ложноотрицательный результат). Тест сдали 10 заболевших человек. Какова вероятность того, что заболевание будет выявлено не менее, чем у 8 человек? 


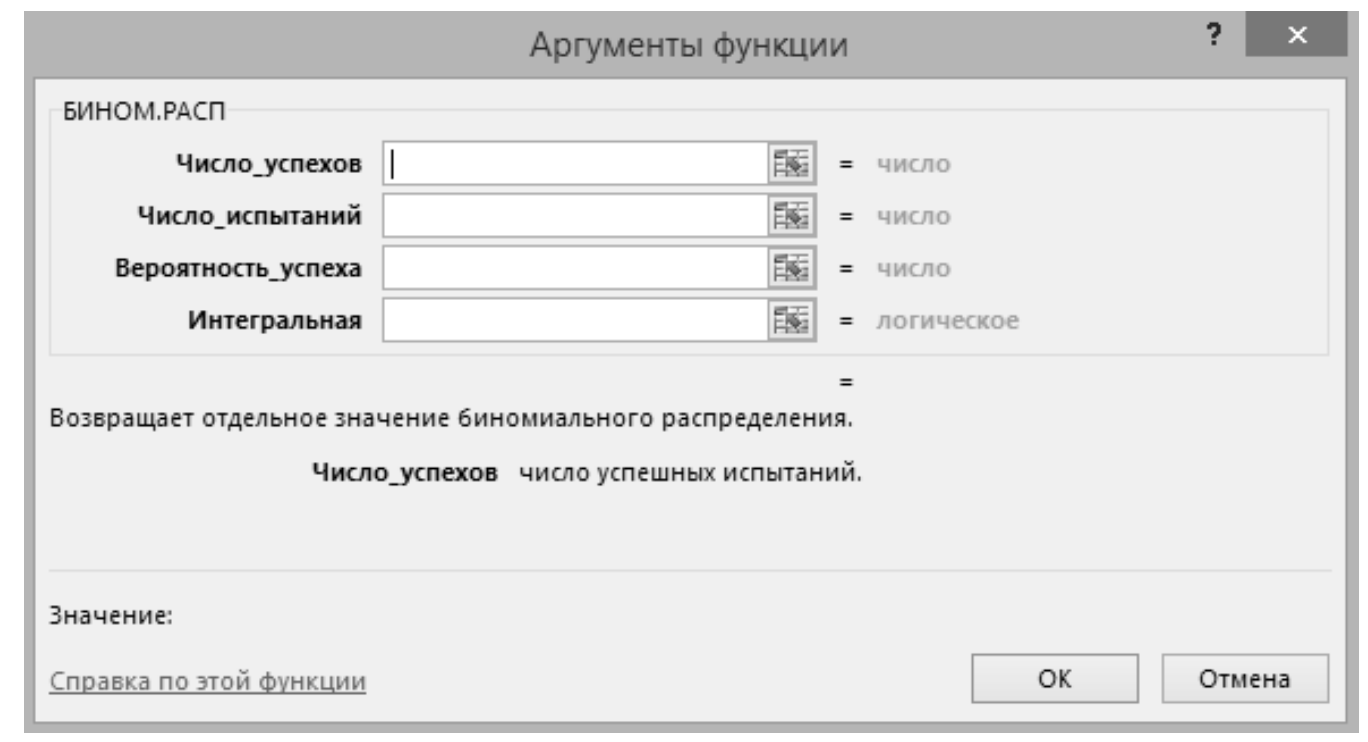

Рис. 2. Аргументы функции «БИНОМ.РАСП»

\begin{tabular}{|c|c|c|c|c|c|}
\hline \multicolumn{4}{|c|}{ Аргументы функции } & $?$ & $\times$ \\
\hline \multicolumn{6}{|l|}{ БИНОМ.РАСП.ДИАП } \\
\hline Испытания & 10 & 監 & \multicolumn{3}{|l|}{$=10$} \\
\hline Вероятность_успеха & 0,8 & 䦨 & \multicolumn{3}{|l|}{$=0,8$} \\
\hline Число_успехов & 8 & 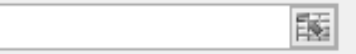 & \multicolumn{3}{|l|}{$=8$} \\
\hline Число_успехов2 & 10 & 臨 & \multicolumn{3}{|l|}{$=10$} \\
\hline \multicolumn{6}{|c|}{$=0,677799526$} \\
\hline \multicolumn{6}{|c|}{ Возвращает вероятность результата испытания с использованием биномиального распределения. } \\
\hline \multicolumn{2}{|c|}{ Число_успехов2 } & \multicolumn{4}{|c|}{$\begin{array}{l}\text { если этот параметр указан, функция возвращает вероятность того, } \\
\text { что число успешных испытаний будет находиться в диапазоне между } \\
\text { значениями число_успехов и число_успехов2. }\end{array}$} \\
\hline \multicolumn{6}{|l|}{ Значение: 0,67780} \\
\hline \multicolumn{2}{|l|}{ Справка по этой функции } & & OK & \multicolumn{2}{|c|}{ Отмена } \\
\hline
\end{tabular}

Рис. 3. Аргументы функции «БИНОМ.РАСП.ДИАП»

Так как формула Бернулли является достаточно простой и понятной, то нет смысла повторять рутинные вычисления, поэтому для автоматизации расчетов целесообразно воспользоваться функцией БИНОМ.РАСП, которая имеет четыре аргумента (Рис. 2):

Суть первых трех аргументов понятна из их названия. Четвертый аргумент - Интегральная - определяет вид функции распределения: интегральная (аргумент Интегральная =1) или весовая (аргумент Интегральная $=0)$. Понятие весовой функции соответствует понятию плотности (или дифференциальной функиии) распре- деления для непрерывных случайных величин, и понятию ряда распределения для дискретных случайных величин. Схема Бернулли задает биномиальное распределение случайных величин, которое является дискретным, о чем мы поговорим более подробно в рамках третьего вопроса нашего занятия. Для решения рассматриваемой задачи можно воспользоваться как функцией распределения, так и рядом распределения.

B Excel 2013 и выше имеется функция БИНОМ.РАСП. ДИАП, которая позволяет решить данную задачу в одно действие (Рис. 3): 


\begin{tabular}{|c|c|c|}
\hline \multirow{2}{*}{} & $\begin{array}{c}\text { Число полож. } \\
\text { тестов }\end{array}$ & Вероятность \\
\hline не более & 7 & 0,322 \\
\hline не более & 10 & 1 \\
\hline & Hе менее 8: & 0,678 \\
\hline
\end{tabular}

Рис. 4. Полная группа событий

\begin{tabular}{|c|c|c|c|c|}
\hline$x$ & 0 & 1 & 2 & 3 \\
\hline \multirow[t]{5}{*}{$p$} & 0,008 & 0,096 & 0,384 & 0,512 \\
\hline & $\begin{array}{c}\text { Числовая } \\
\text { хар-ка }\end{array}$ & $\begin{array}{c}\text { Формула } \\
\text { бином расп. }\end{array}$ & $\begin{array}{c}\text { Проверка } \\
\text { по типовой } \\
\text { формуле }\end{array}$ & \\
\hline & $M(X)$ & & 2,40 & \\
\hline & $D(\mathrm{X})$ & & 0,48 & \\
\hline & $\sigma$ & & 0,69 & \\
\hline
\end{tabular}

Рис. 5. Ряд распределения

Однако на данном этапе для понимания сущности теоретико-вероятностных подходов более полезным является использование функции БИНОМРАСП.

Рассмотрим решение этой же задачи, используя функиию распределения. Для этого вспомним, что функцией распределения случайной величины $X$ называется функция $F(x)$, задающая вероятность того, что случайная величина $X$ примет значение меньшее $x$, т.е.

$$
F(x)=P(X<x)
$$

Также вспомним, что следующие свойства функции распределения:

$$
\lim _{x \rightarrow \infty} F(x)=1 ; P(a \leq x<b)=F(b)-F(a) .
$$

Тогда для рассматриваемой задачи будем иметь (вероятность того, что случайная величина примет значение не менее 8):

$$
P(x \geq 8)=1-P(x<8)=1-F(8) .
$$

Однако надо учесть, что при использовании функции БИНОМРАСП с аргументом Интегральная = 1 выполняется выражение $F(x)=P(X \leq x)$, т.е. вероятность того, что случайная величина $X$ примет значение не более $x$, поэтому в нашем случае в качестве левой границы диапазона надо использовать значение 7, так как биномиальное распределение является дискретным.
События $X \leq 7$ и $X \geqslant 8$ образуют полную группу событий ( $X$ является дискретной случайной величиной, поэтому границы областей не совпадают, Рис. 4), поэтомy:

$$
P(x \leq 7)+P(x \geq 8)=1 .
$$

Дискретная случайная величина, принимающая целые неотрицательные значения с вероятностью, определяемой по формуле Бернулли, называется распределенной по биномиальному закону.

Математическое ожидание и дисперсия биномиального распределения имеют вид:

$$
M(x)=n p ; D(x)=n p(1-p) .
$$

ПОЗ № 3. Постройте ряд распределения числа выявленных заболеваний по результатам решения ПОЗ № 1. Найдите математическое ожидание, дисперсию и среднее квадратическое отклонение по формулам для биномиального распределения.

Значения в первую таблицу заносятся вручную с листа ПОЗ № 1. Затем вводятся формулы для расчета числовых характеристик (Рис. 5).

После построения ряда распределения будут автоматически построены гистограмма и полигон распределения (Рис. 6). 
Гистограмма и полигон биномиального распределения

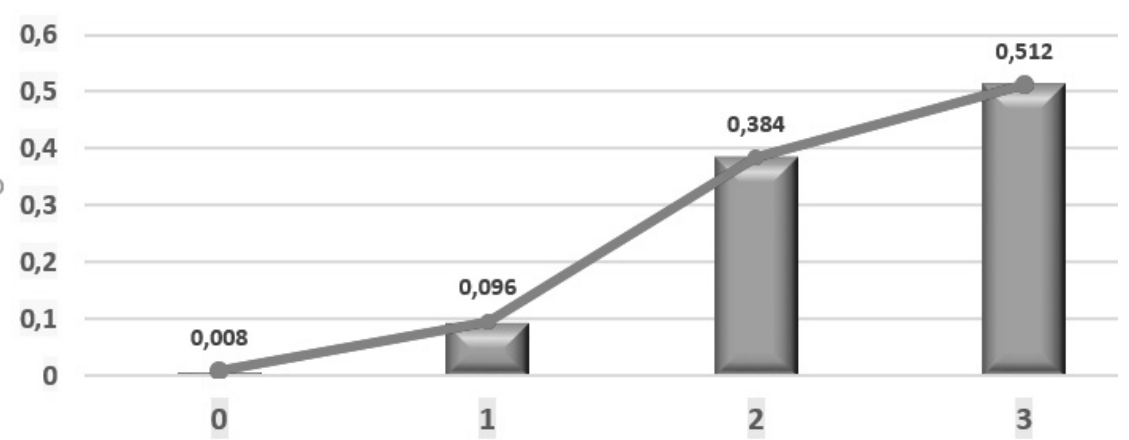

Рис. 6. Гистограмма и полигон биномиального распределения

\begin{tabular}{c|c|c|}
8 & \multicolumn{3}{|c}{ 1. Нахождение числовых характеристик случайной величины } \\
\hline 9 & $\mid \begin{array}{c}\text { Числовая } \\
\text { хар-ка }\end{array}$ & $\begin{array}{c}\text { Формула } \\
\text { бином расп. }\end{array}$ \\
\hline 10 & $\mathbf{M}(\boldsymbol{X})$ & 30,00 \\
\hline 11 & $\boldsymbol{D}(\mathbf{X})$ & 29,10 \\
\hline 12 & $\boldsymbol{\sigma}$ & 5,39 \\
\hline
\end{tabular}

Рис. 7. Нахождение числовых характеристик случайной величины

Построенное биномиальное распределение является ассиметричным, так как $p=0,8$. При $p=0,5$ распределение является симметричным.

При $p \neq 0,5$ распределение приближается к симметричному при увеличении числа испытаний $n$. Это приближение будет происходить тем быстрее, чем ближе значение $p$ к 0,5.

Кроме того, при увеличении $n$ биномиальное распределение можно аппроксимировать нормальным распределением с теми же математическим ожиданием и дисперсией.

ПОЗ № 4. Выдвинуто предположение, что 3\% населения города являются зараженными. При тестировании, проводимой мобильной лабораторией, случайным образом были взяты пробы у 1000 жителей. Какова вероятность того, что в протестированной группе окажется не менее 20 зараженных при условии полной достоверности теста? Решите задачу, используя нормальное и биномиальное распределения.

Рассматриваемая задача по существу эквивалентна проведению 1000 испытаний Бернулли с вероятностью успеха при одном испытании 0,03.
Для аппроксимации биномиального распределения нормальным найдем его числовые характеристики:

$$
\begin{aligned}
& M(x)=n p=1000 \times 0,03=30 ; \\
& D(x)=n p(1-p)=1000 \times 0,03 \times 0,97=29,1 ; \\
& \sigma=\sqrt{D(x)}=5,39 .
\end{aligned}
$$

Так как функция нормального распределения является непрерывной, а функция биномиального распределения - дискретной, то для аппроксимации дискретного интервала между точками 19 и 20 биномиального распределения возьмем значение 19,5 для нормального распределения.

Для нормального распределения, так как оно является непрерывным, имеем:

$$
P(x \geq 19,5)=P(x>19,5)=1-F(19,5) .
$$

Используем найденные характеристики в качестве аргументов функции НОРМРАСП:

Рассчитаем искомую вероятность через биномиальное распределение, при этом надо помнить, что это 


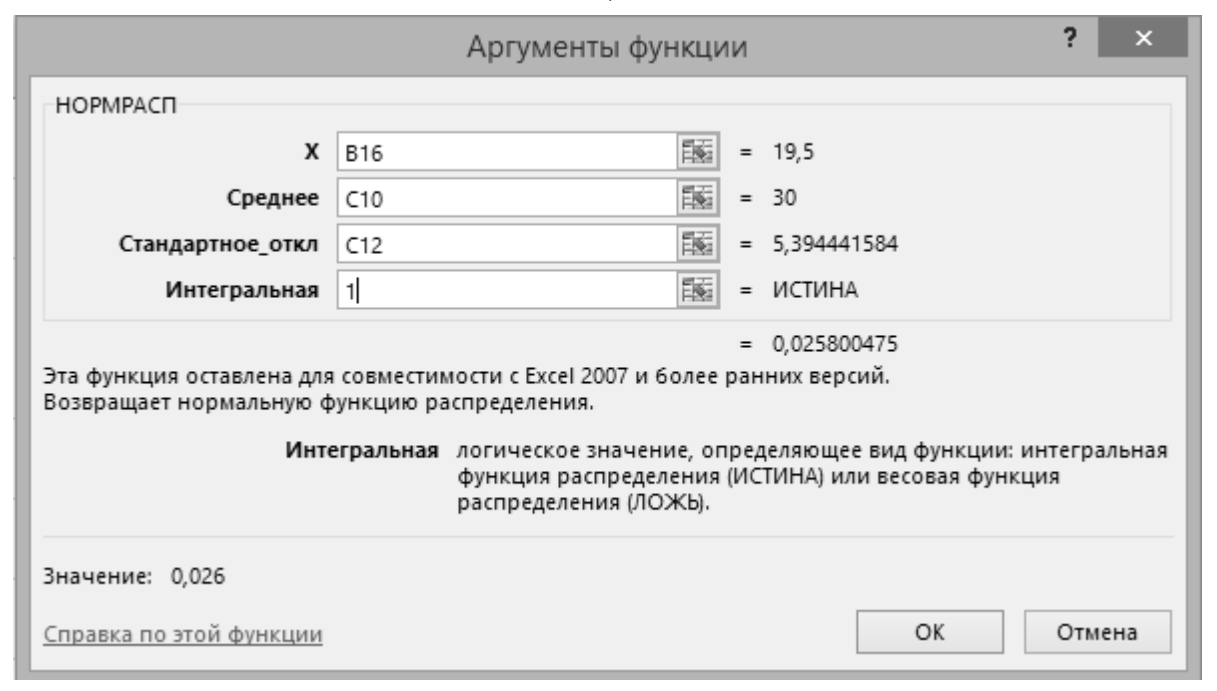

Рис. 8. Аргументы функции «НОРМРАСП»

\begin{tabular}{|c|c|c|c|}
\hline 15 & & $\begin{array}{c}\text { Число } \\
\text { зараженных }\end{array}$ & Вероятность \\
\hline 16 & не более & 19,5 & 0,026 \\
\hline 17 & не более & 1000 & 1,000 \\
\hline 18 & \multicolumn{2}{|r|}{ Не менее 19,5: } & 0,974 \\
\hline
\end{tabular}

Рис. 9. Расчет вероятности через нормальное распределение

\begin{tabular}{|l|l|c|c|}
\hline 20 & \multicolumn{3}{|c}{ 3. Расчет вероятнсти через биномиальное распределение } \\
\hline 21 & $\begin{array}{c}\text { Число } \\
\text { зараженных }\end{array}$ & Вероятность \\
\hline 22 & не более & 19 & 0,020 \\
\hline 23 & не более & 1000 & 1,000 \\
\hline 24 & He менее 20: & 0,980 \\
\hline
\end{tabular}

Рис. 10. Расчет вероятности через биномиальное распределение

распределение является дискретным. Воспользуемся известной нам функцией БИНОМРАСП:

Как видим, при большом числе испытаний $n$ биномиальное распределение даже при $p$, существенно отличающимся от 0,5, достаточно точно аппроксимируется нормальным распределением.
Использование пакетов прикладных программ в условиях цифровизации математического образования способствует повышению уровня математической подготовки будущих специалистов МЧС России, а также формированию и развитию профессиональной мотивации. 


\section{ЛИТЕРАТУРА}

1. Artamonov V.S., Ivanov A.Y., Sharapov S.V., Trofimets E.N., Trofimets V. Ya. Information systems and processes in the analytical training of management scholars // Espacios, Vol. 38 (N25), 2017, P. 18.

2. Батьковский А.М., Трофимец В.Я. Системы поддержки принятия решений с модулями прикладных математических моделей и методов // Вопросы радиоэлектроники. - 2015.- № 9.- - С. 253-275.

3. Трофимец В.Я. Оптимизация в ЕхсеІ: учеб. пособие / В.Я. Трофимец, Е.Н. Трофимец.- Ярославский государственный технический университет, Ярославль, 2008. - 104 с.

4. Трофимец Е.Н. Математическое и программное обеспечение информационных систем поддержки принятия решений / Монография под общ. ред. Э.Н. Чижикова.— СПб.: Санкт-Петербургский университет ГПС МЧС России, 2019. - 174 с.

5. Трофимец Е.Н., Трофимец В.Я. Компьютерное моделирование в математическом образовании инженеров // Сборник научных трудов III Всероссийской науч. конф. «Современные образовательные технологии в подготовке специалистов для минерально-сырьевого комплекса», 5-6 марта 2020 г. Санкт-Петербург: Санкт-Петербургский горный университет, 2020. - С. 351-357.

6. Трофимец Е.Н. Современная цифровая образовательная среда в процессе изучения математических дисциплин // Сборник трудов международной научной конференции «Наука. Исследования. Практика». 26 августа 2019 г.— Санкт-Петербург. СПб: ГНИИ «Нацразвитие», 2019.— С. 48-50.

7. ТрофимецЕ.Н. Квопросу совершенствования информационно-аналитической подготовки специалистов МЧС России // Сборник трудов международной научной конференции «Наука. Исследования. Практика». 26 августа 2019 г. — СПб: ГНИИ «Нацразвитие», 2019. — С. 50-53.

(c) Трофимец Елена Николаевна ( ezemifort@inbox.ru ).

Журнал «Современная наука: актуальные проблемы теории и практики»

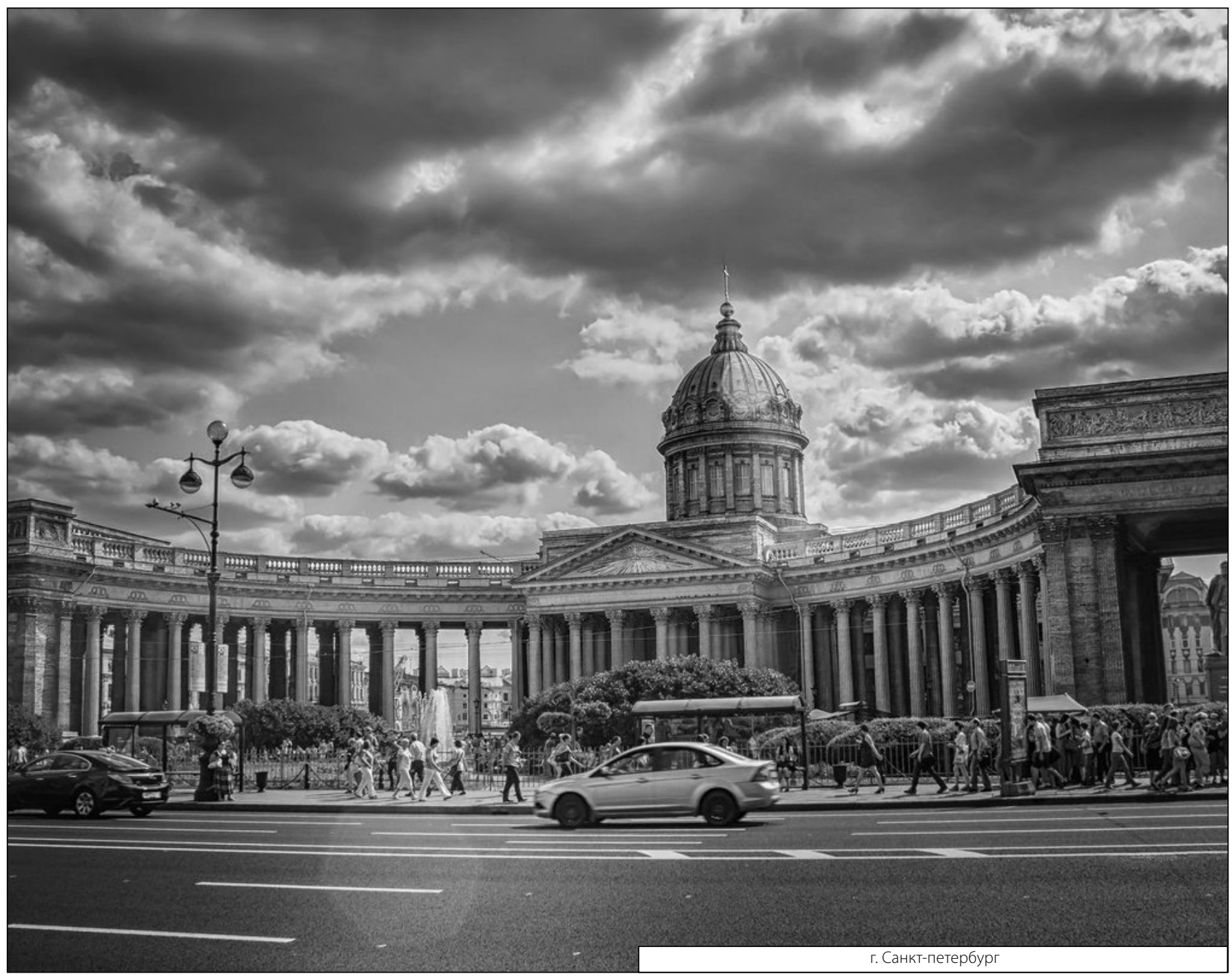

